\title{
DeepBBWAE-Net: A CNN-RNN Based Deep SuperLearner For Estimating Lower Extremity Sagittal Plane Joint Kinematics Using Shoe-Mounted IMU Sensors In Daily Living
}

This paper was downloaded from TechRxiv (https://www.techrxiv.org).

\section{LICENSE}

CC BY 4.0

SUBMISSION DATE / POSTED DATE

23-07-2021 / 26-07-2021

\section{CITATION}

Hossain, Md Sanzid Bin; Drantez, Joseph; Choi, Hwan; Guo, Zhishan (2021): DeepBBWAE-Net: A CNN-RNN Based Deep SuperLearner For Estimating Lower Extremity Sagittal Plane Joint Kinematics Using ShoeMounted IMU Sensors In Daily Living. TechRxiv. Preprint. https://doi.org/10.36227/techrxiv.15040653.v1

$\mathrm{DOI}$ 


\title{
DeepBBWAE-Net: A CNN-RNN Based Deep SuperLearner For Estimating Lower Extremity Sagittal Plane Joint Kinematics Using Shoe-Mounted IMU Sensors In Daily Living
}

\author{
Md Sanzid Bin Hossain, Student Member, IEEE Joseph Dranetz, Student Member, IEEE and Hwan Choi, \\ Member, IEEE, Zhishan Guo Senior Member, IEEE
}

\begin{abstract}
Measurement of human body movement is an essential step in biomechanical analysis. The current standard for human motion capture systems uses infrared cameras to track reflective markers placed on the subject. While these systems can accurately track joint kinematics, the analyses are spatially limited to the lab environment. Though Inertial Measurement Unit (IMU) can eliminate the spatial limitations of the motion capture system, those systems are impractical for use in daily living due to the need for many sensors, typically one per body segment.

Due to the need for practical and accurate estimation of joint kinematics, this study implements a reduced number of IMU sensors and employs machine learning algorithm to map sensor data to joint angles. Our developed algorithm estimates hip, knee, and ankle angles in the sagittal plane using two shoe-mounted IMU sensors in different practical walking conditions: treadmill, level overground, stair, and slope conditions. Specifically, we proposed five deep learning networks that use combinations of Convolutional Neural Networks (CNN) and Gated Recurrent Unit (GRU) based Recurrent Neural Networks (RNN) as base learners for our framework. Using those five baseline models, we proposed a novel framework, DeepBBWAE-Net, that implements ensemble techniques such as bagging, boosting, and weighted averaging to improve kinematic predictions. DeepBBWAE-Net predicts joint kinematics for the three joint angles under all the walking conditions with a Root Mean Square Error (RMSE) 6.93-29.0\% lower than base models individually. This is the first study that uses a reduced number of IMU sensors to estimate kinematics in multiple walking environments.
\end{abstract}

Index Terms-Kinematics Estimation; Wearable IMU Sensors; Ensemble Learning; Deep Learning; Machine Learning

\section{INTRODUCTION}

Human gait is the complex and coordinated movement of the limbs which allows for locomotion. Gait motion analysis is a qualitative and quantitative tool used to assess people's kinesiological health. For example, as people age, they experience transformations in their gait pattern [1]. Early detection of this transformation is useful in assessing fall risk among the elderly. Gait abnormalities can also occur due to neurological damage related to traumatic injury or disease [2]. As gait alterations can significantly impact quality of life, gait analysis has become a more popular and relevant research topic. Gait motion assessment can aid in the evaluation of the severity, progression, and diagnosis of a disease or injury. Joint kinematics are necessary spatiotemporal measures to assess abnormal gait function

Md Sanzid Bin Hossain is with the Electrical and Computer Engineering Department, University of Central Florida, Orlando, FL 32816, USA (e-mail: sanzid@knights.ucf.edu)

Joseph Dranetz is with the Mechanical and Aerospace Engineering Department, University of Central Florida, Orlando, FL 32816, USA (email: jmdranetz@knights.ucf.edu)

Hwan Choi is with the Mechanical and Aerospace Engineering Department, University of Central Florida, Orlando, FL 32816, USA (e-mail: hwan.choi@ucf.edu)

Zhishan Guo is with the Electrical and Computer Engineering Department, University of Central Florida, Orlando, FL 32816, USA (e-mail: zsguo@knights.ucf.edu) in the clinical setting. For example, knee joint kinematics have been used to evaluate hypermobility syndrome (HMS) in children [3]. In addition to disease assessment, gait motion analysis is also a valuable tool in the field of sports medicine [4].

Traditional movement measurement techniques for gait assessment use 3D infrared light motion capture cameras to measure the trajectories of reflective markers placed on the human body. The acquired data is then processed with dynamic analysis software to compute joint angles [OpenSim, Visual3D, Vicon Nexus]. Although this method is regarded as the ground truth measure for dynamic function of human motion [5], it requires manual data processing, expert device operation and only works in a spatially and temporally restricted area due to the need for a large number of cameras to define a limited capture volume. It has been suggested that the use of Inertial Measurement Unit (IMU) sensors may overcome some of the disadvantages with current methods for assessing the dynamic function of human motion. Recent studies have shown that IMU's can be used to calculate joint angles [6]. Researchers can estimate human movements outside of the laboratory environment by replacing traditional motion capture cameras with wearable IMU sensors. These techniques also enable potential applications in long-term use and integrative daily monitoring. However, the use of IMU's requires setup before collecting motion data. For example, each IMU sensor needs to be calibrated for each specific body segment as the position and orientation of every sensor assigned to each segment must be defined. While several calibration processes have been proposed [7], [8], they are prone to error as they are challenging to implement onto the subject consistently. Traditional IMU-based estimation also requires the placement of sensors onto each segment of the body, making them too cumbersome for application in daily/continuous monitoring. We believe that significant redundancy occurs in a multisensor human motion capture system such that it is possible to reduce the number of sensors without a significant loss in kinematic accuracy.

Challenges. The key challenge to estimating joint angles using a reduced number of sensors is the lack of input signal. Human movement is complex in nature [9], and it is challenging to infer several joint angles using only sensors at the feet. However, it is possible to infer the missing IMU signals virtually by utilizing statistic methods and signal processing techniques [10]-[12] while using a reduced number of sensors. These statistical methods and signal processing techniques are resource-intensive and impractical for application in real-time gait monitoring. A machine learning model may replace these statistical methods and signal processing techniques to allow for real-time estimations of kinematics.

As of late, there has been a massive wave of innovations in machine learning. A properly-trained model can estimate kinematics with decent accuracy. Machine learning models implementing Gated Recurrent Unit (GRU) based Recurrent Neural Networks (RNN), or Convolutional Neural Networks (CNN) can extract relevant features from the raw data of a reduced set of IMU sensors to estimate 
kinematics. As a result, these machine learning models can help overcome the limitations introduced by static pose calibration, biomechanical modeling complexity, magnetometer data error, and multisensor constraints.

Ensemble learning is a classical machine learning approach that has created significant attention among researchers in recent decades. The attention is well deserved as it yields significant performance improvement in many real-world applications. It is the technique of combining multiple weak learners in an intelligent way to create a stronger learner, which has better predictive performance than those single weak learners individually. There are many well-established ensemble learning methods: bagging, boosting, stack generalization, weighted average ensemble, etc. In some of these methods, training data is manipulated, passed to a homogeneous learner, and then combined using averaging or majority voting for regression and classification problems, respectively. In other methods, parameters or the structures of the models are varied to create multiple learners and then combined using either a secondary learner called a meta-learner or a weighted averaging system to get the final ensemble model's prediction. To get accurate kinematic estimations in daily living, a combination of different ensemble approaches may be helpful.

Contribution. This work aims to estimate kinematics (sagittal plane hip, knee, and ankle joint angles) under various walking conditions (stair, slope, level overground, and treadmill) using just two IMU sensors (one per shoe). We propose five deep learning models consisting of Convolutional Neural Networks (CNN) and Gated Recurrent Unit (GRU) based Recurrent Neural Networks (RNN) for feature exaction, classification, and time-series data predictions. These models are well suited to process raw IMU data. We further develop a novel CNNRNN based deep super learner model, Deep Bagging, Boosting, and Weighted Average Ensemble (DeepBBWAE-Net). This super learner implements various traditional ensemble methods (bagging, boosting, weighted average ensemble) to combine the deep learning neural networks. Combining predictions from multiple models improves the accuracy of the joint angle estimation. Our experimental results suggest that the proposed super learner method outperforms each of the learners individually. Furthermore, DeepBBWAE-Net uses raw data as the input of the neural network without any feature extraction or time normalization, and it will help minimize the system's time complexity, paving the way for real-time gait monitoring.

The rest of this paper is organized as follows: Section II briefly reviews recent literature about machine learning-based kinematics estimation using IMU and fundamental machine learning concepts that are used in the construction of the DeepBBWAE-Net framework. The problem statement and the structures of the DeepBBWAE-Net framework, as well as its base learners, are discussed in Section III. Section IV describes the procedures followed for data collection, the data pre-processing, the parameters chosen for the machine learning model, the statistical validation methods, and results of the prediction. Section V discusses implications from these results, describes study limitations, and proposes future work. We conclude the paper in Section VI.

\section{Related Work}

This section will discuss the current state-of-the-art machine learning methods for IMU-based kinematics estimation and traditional neural network components used in our framework. First, we will discuss studies where a complete set of IMU sensors was used to estimate the kinematics. Then we will discuss attempts to employ a reduced number of IMU sensors to achieve similar kinematic predictions. After that, we will discuss the literature related to traditional machine learning techniques used in our framework.

\section{A. IMU and Machine learning-based approach}

Mundt et al. [13] used five IMU sensors to estimate 3D joint kinematics. They segmented the gait cycle based on the algorithm proposed by Maiwald et al. [14]. They normalized their time-series data onto the gait cycle before inputting it into the feed-forward neural network. In [15]. [16], the author used simulated IMU sensor data for 3D kinematic prediction. In [17], a simulation technique with a musculoskeletal model was used to augment the data set used to train an algorithm to predict kinematics for running and walking. Data from four IMU sensors was used to track the kinematics of a single leg. In [18], five IMU sensors were used to estimate kinematics in walking and running on a treadmill. More recently, in [19], a combined deep learning and optimization framework was proposed to estimate 3D kinematics using seven IMU sensors for walking and running. These studies have used a relatively large set of sensors and were only conducted on treadmill or overground conditions.

\section{B. Reduced Sensor-Based Approach}

Many studies have employed machine learning for kinematics estimation of treadmill and overground walking conditions [13], [15], [16], [18], [17], [19] using a large number of sensors. Due to the impracticality associated with using a full set of IMU sensors, a limited number of studies have employed a reduced set of IMU sensors to estimate joint kinematics. Lim et al. [20] used a single IMU mounted on participants' sacrum to estimate kinetics and kinematics. They processed the IMU data to calculate acceleration, velocity, and displacement over time and used this data as input for an artificial neural network. This data processing was done retroactively, and the pre-processing they performed may have resulted in an additional error. The method was also not able to estimate kinetics and kinematics for both legs simultaneously. Gholami et al. [21] proposed a single accelerometer approach for calculating the kinematics of running on a treadmill. As their machine learning model uses input data from past and future samples for present kinematics prediction, it will not make predictions for the start and the end portion of the data. As they have built their model based on treadmill data, a repetitive process, the predictions are good as it is easier to predict repetitive kinematics when both past and future data are provided. But in an uncontrolled environment like stair and slope conditions, their predictive model may not perform well as there is significant variability in those conditions. More recently, [22] used a single sensor on the foot to estimate kinematics during overground walking. They used gait cycle normalization, a Hilbert-Huang transformation, to process the data making it difficult for real-time prediction due to the longer computation time. Using a single sensor on the foot may create ambiguity when the foot is in full contact with the ground because the IMU's shouldn't record any accelerations or angular velocity, but the hip, knee, and ankle angles are still changing.

\section{Fundamental Neural Network Components and Methodologies}

This study aims to use machine learning techniques to estimate lower extremity joint angles as accurately as possible. Using an effective machine learning model is an essential step for this purpose. In this subsection, we will discuss the theoretical details of the methods used to build DeepBBWAE-Net and justify their use.

Gated Recurrent Unit (GRU). Long-short term memory (LSTM) is a special type of Recurrent neural network (RNN) that can capture time-dependency in time series data without suffering from the gradient vanishing or explosion problem [23], and thus can process longer sequences of data (compared to regular RNN). Nevertheless, LSTM is heavily parameterized and takes a longer time to train. Cho 
et al. [24] first proposed Gated Recurrent Units (GRU), a special case of LSTM without an output gate, resulting in fewer parameters and a reduction in the required training time. GRU will be used for feature learning from the accelerometer and gyroscope time-series data in our study.

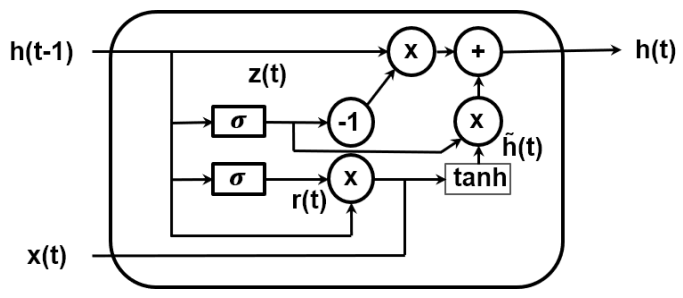

Fig. 1. GRU cell

Specifically, two gates are introduced in the GRU cell unit: the reset gate and the forget gate. The update gate determines what new information has to be added or dropped from previous memory. The reset gate decides how much previous information needs to be forgotten. The relationship between input and output of the hidden units of the GRU is given below:

$$
\begin{array}{r}
r(t)=\sigma\left(W_{r} x(t)+V_{r} h(t-1)+b_{r}\right) \\
z(t)=\sigma\left(W_{z} x(t)+V_{z} h(t-1)+b_{z}\right) \\
\tilde{h}(t)=\tanh \left(W_{c} x(t)+V_{c}(r(t) \odot h(t-1))\right) \\
h(t)=(1-z(t)) \odot h(t-1)+z(t) \odot \tilde{h}(t)
\end{array}
$$

where $\mathrm{r}(\mathrm{t})$ is the reset gate, $\mathrm{z}(\mathrm{t})$ is the update gate, and $W, V, b$ are parameters of the model. $\odot$ represents the element wise dot product. Convolutional Neural Networks (CNN). CNN's are computationally optimized feedforward deep neural networks with sparse convolutional layers. Each convolutional layer has kernel functions that convolute small patches of the input signal. This makes convolutional networks more optimized for feature extraction and less computationally expensive. They have been successfully applied to gait recognition tasks where the input signal is an accelerometer and gyroscope time-series data [25], [26]. The use of pre-processed data from the convolutional network is advantageous as handcrafted features extracted by humans may not sufficiently represent the underlying relations. CNN models can use raw data from the input to learn their feature representation through backpropagation while maximizing the best mapping of the IMU data to the joint kinematic data.

Bagging. Bootstrap aggregating (bagging) is a classic ensemble machine learning technique proposed by Breiman [27] which can be applied in both classification and regression problems. Bootstrap samples are created using random sampling with replacement from a data set. Typically, a bootstrap sample size is less than or equal to the size of the original data set from which it was created. Each bootstrap data subset is then passed to a homogeneous learner to create multiple distinct trees, which can be aggregated by voting or averaging for classification and regression problems, respectively. Bagging reduces the variance of prediction and increases the stability and accuracy of the machine learning model.

Boosting. In bagging, multiple models are trained independently, while in boosting, multiple weak learners are trained sequentially in an adaptive way to form a stronger learner. To reduce bias during the learning process, models adaptively give more weight to the instances in the data set that were weakly interpreted by previous models (and less weight to cases of successful interpretations). We implement a Gradient Boosting Machine (GBM) [28], which can be considered an optimization process toward finding a model that minimizes loss. With a GBM, a base learner (decision tree) is trained on the residual of the previous decision tree to make its prediction. Multiple models are then aggregated after convergence criteria are fulfilled. In GBM, to avoid overfitting, the learning rate is used to shrink the residual prediction, causing variance reduction.

Weighted Average Ensemble (WAE). Model averaging is another ensemble approach where predictions from each model are aggregated to generate a final prediction in a regression problem. Different weights are applied to different models according to their effectiveness toward the prediction to ensure optimal performance from the final ensemble model. Given $N$ number of models and the output from each model $Y_{1}, Y_{2}, \ldots, Y_{N}$, the output of the weighted ensemble model is:

$$
Y=\left[\sum_{k=1}^{N} W_{k} Y_{k}\right]
$$

Where $W_{k}$ can be found with any convex optimization approach, which calculates the optimal weight for each model to maximize the performance of the final ensemble model.

Convex Optimization. Sequential least-square programming (SLSQP) is a gradient-based optimization method, which was first proposed by Kraft and Schnepper [29] by modifying the least square methods of Lawson and Hanson [30]. The optimizer uses the Han-Powell quasi-Newton method [31] with a BroydenFletcher-Goldfarb-Shanno (BFGS) algorithm to update the B-matrix and L1-test function. In our framework, we use this optimization procedure to find the optimal weights of the models and to minimize the RMSE between ground truth and the predictions.

\section{Proposed Approach}

In this section, we will discuss the problem statement, detailed workflow of DeepBBWAE-Net Framework, and base learners used for building the framework.

\section{A. Problem Statement}

The purpose of the study is to estimate joint angles of the hip, knee, and ankle using two shoe-mounted sensors in different practical walking scenarios: stair, slope, and level overground. To date, no study has attempted to employ a reduced number of sensors to estimate joint angles under multiple conditions. As the number of sensors is reduced, an effective machine learning model is essential to accurate kinematic estimations. For this reason, we proposed a novel framework, DeepBBWAE-Net, which leverages bagging, boosting, and weighted average ensemble techniques to improve the prediction performance. We believe two sensors should be the minimum necessary number and thus set this limit in our design. A moment's thought should convince the reader that when one of the feet is in full contact with the ground, its IMU signal becomes zero, and the sensor on the other foot can provide the network with the necessary information to extract kinematics of both legs.

\section{B. DeepBBWAE-Net Framework}

The DeepBBWAE-Net framework consists of three fundamental blocks: BagBoost, Weighted Average Ensemble (WAE), and BaseBoost (Fig. 2). The BagBoost block consists of multiple BagBoost cells. The function of each BagBoost cell is to apply bagging and boosting to each base learner to improve the prediction performance. The function of the BaseBoost block is to generate boosted predictions of the base learner using the whole data set. This block gives additional information to the framework for improving the prediction performance. The function of the WAE block is to combine the 


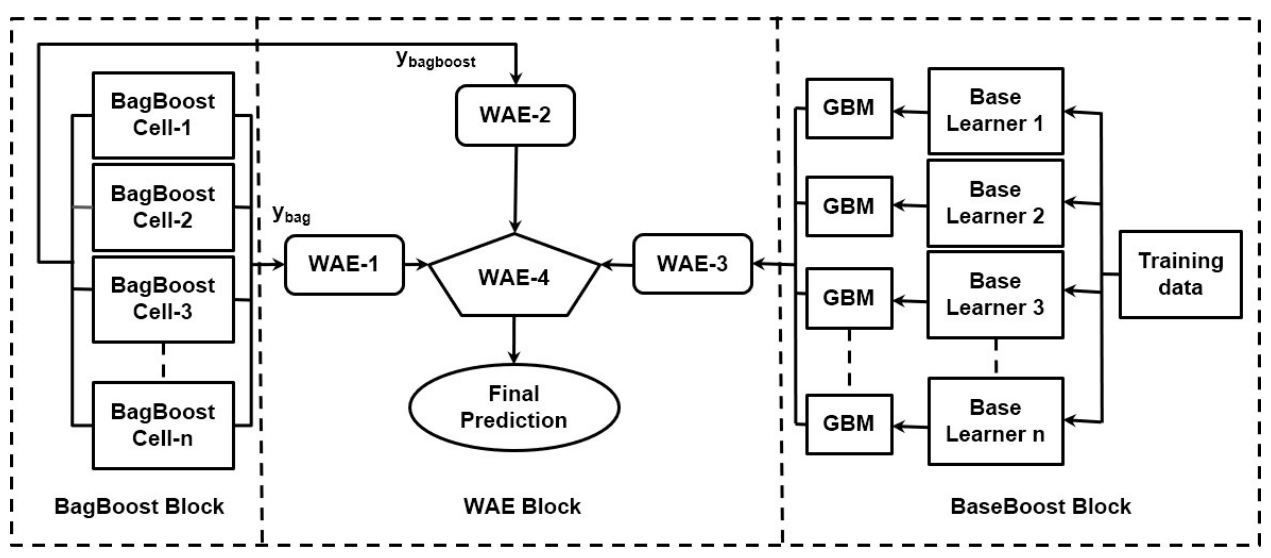

Fig. 2. DeepBBWAE-Net consisting of three blocks- a BagBoost, WAE, and BaseBoost Block

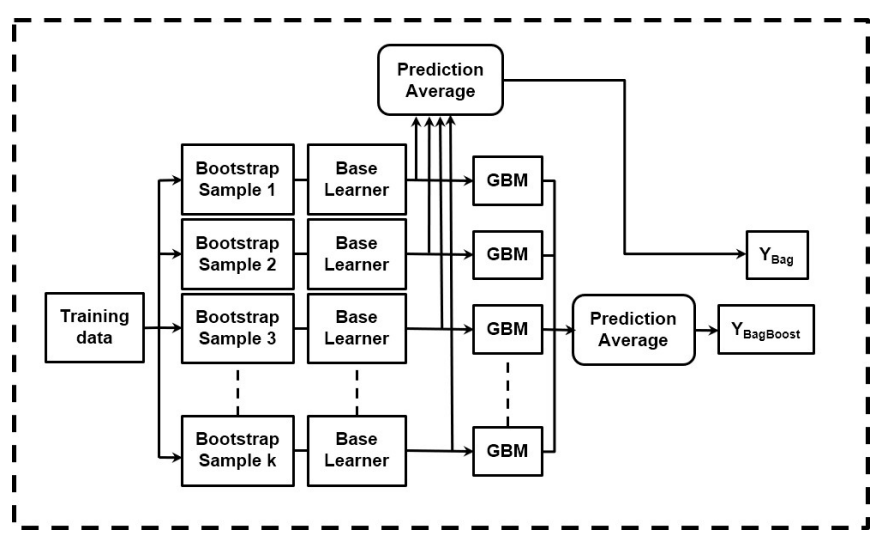

Fig. 3. A BagBoost Cell

outputs of the multiple BagBoost and BaseBoost blocks using optimal weighted averaging to generate the final prediction.

BagBoost block. The BagBoost block consists of multiple BagBoost cells. The number of BagBoost cells (n) will depend on the number of base learners used in the framework. Fig. 3 demonstrates the workflow of a single BagBoost cell. In each cell, a single base learner is used to get two prediction .i.e., $Y_{\text {bag }}, Y_{\text {bagboost }}$. Ensemble learningbagging and boosting (GBM) are used to get these two predictions. At first, $\mathrm{k}$ bootstrap samples are created from the training data, and each sample is used to train the same base learner. Predictions from each base learner are then averaged to get $Y_{b a g}$. Additionally, each $Y_{b a g}$ is passed to a GBM, trained with the validation data, to increase generalization. The prediction from each GBM learner is then averaged to get $Y_{\text {bagboost }}$.

BaseBoost block. Unlike in the BagBoost cells, the entire training data set is used to train the base learners in the BaseBoost block. To further improve the performance, results from the base learners are passed to a GBM, trained with the validation data, and has an output named $Y_{\text {baseboost }}$.

WAE block. In the WAE block, we have four WAE cells: WAE-1, WAE-2, WAE-3, WAE-4. The function of each cell is to combine results from multiple branches to generate a better prediction.

In WAE-1, prediction $Y_{b a g}$ from the BagBoost cells are used to generate Bag-WAE-Net. In WAE-2, prediction $Y_{\text {bagboost }}$ from the BagBoost cells are used to create BagBoost-WAE-Net. In WAE-3, prediction $Y_{\text {baseboost }}$ from the BaseBoost cells are used to generate BaseBoost-WAE-Net. WAE-1, WAE-2, and WAE-3 are then used to build WAE-4, which produces the final prediction. To find the optimal weights in the WAE block, we used a convex optimization method, the SLSQP optimization algorithm, where the loss function was defined as the RMSE between ground truth kinematic data and the kinematic predictions.

\section{Base learners}

This subsection will discuss the details of the five base learners that we have developed to build DeepBBWAE-Net. We used several blocks consisting of 1D/2D convolutional layers, Bi-GRU layers, and fully connected layers to create the base learners. First, we will introduce the base learners. Then, we will describe the different fundamental blocks used to create the base learners.

1) Base learner 1: Conv2D-Net: In base learner 1 (Fig. 4), two fundamental blocks, i.e., 2D convolutional and fully connected blocks, were used. First, batch normalization $(\mathrm{BN})$ is applied on the input signal to address the heterogeneity of source data [32] to perform regularization. After batch normalization, a 2D convolutional block and a fully connected block were added consecutively. A flattening layer is added to flatten the output from the fully connected blocks, which is then connected to the last prediction layer.

2) Base learner 2: Bi-GRU-Net: In Base learner 2 (Fig. 8), a BiGRU block is used after batch normalizing the input data. A flattening layer was then added to flatten the output from Bi-GRU block and connected to the final output layer.

3) Base learner 3: Hybrid Conv1D-GRU-Net: Base learner 3 (Fig. 5) consists of a Bi-GRU, a 1D convolutional, and a fully connected block. After batch normalization, features are extracted from the input using the Bi-GRU block. The output of the Bi-GRU block is then passed to a $1 \mathrm{D}$ convolutional block to extract the features further. A fully connected block was added after the 1D convolutional block. The output from the fully connected block was then flattened to connect to the output layer.

4) Base learner 4: Hybrid Conv2D-GRU-Net-1: In base learner 4 (Fig. 6), we concatenated the output from the flattening layer of base learners 1 and 2. Both Conv2D-Net and Bi-GRU-Net were used to extract features from the IMU data. To get advantages from both model's feature extraction, we concatenated features from both of the models. This concatenated layer is then connected to the output layer for prediction.

5) Base learner 5: Hybrid Conv2D-GRU-Net-2: In base learner 5 (Fig. 7), a 2D convolutional block is first used to extract features. A regular flattening layer will flatten the input into a $2 \mathrm{D}$ tensor and reduces the temporal and feature dimensions into a single one. For this reason, we instead used a TimeDistributed wrapper of Keras [33] to flatten the output to produce a 3D tensor, which is the required input of the Bi-GRU layer. With this, a Bi-GRU block was added 
Fig. 4. Base Learner 1

2D Convolutional Block

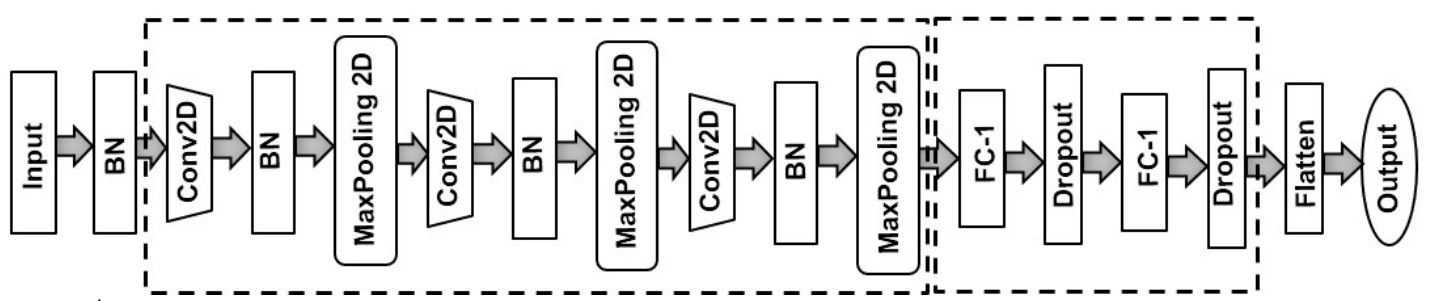

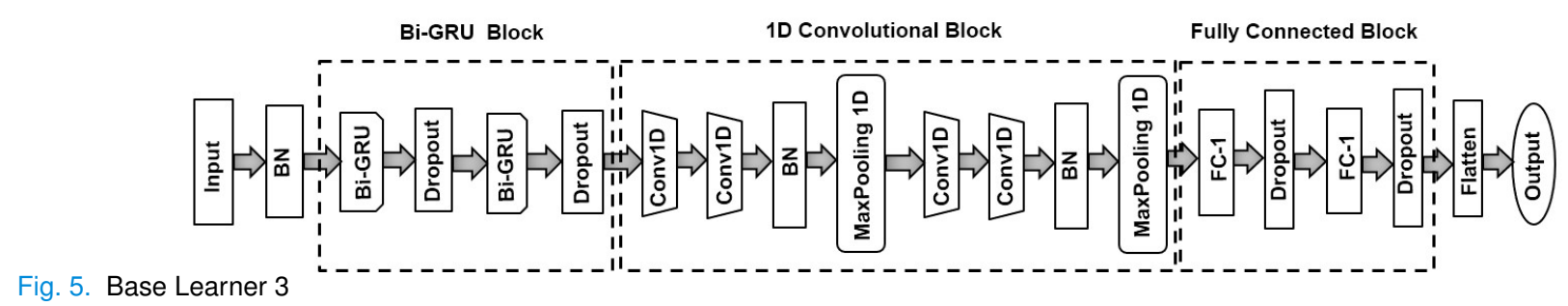

Fig. 5. Base Learner 3

2D Convolutional Block

Fully Connected Block

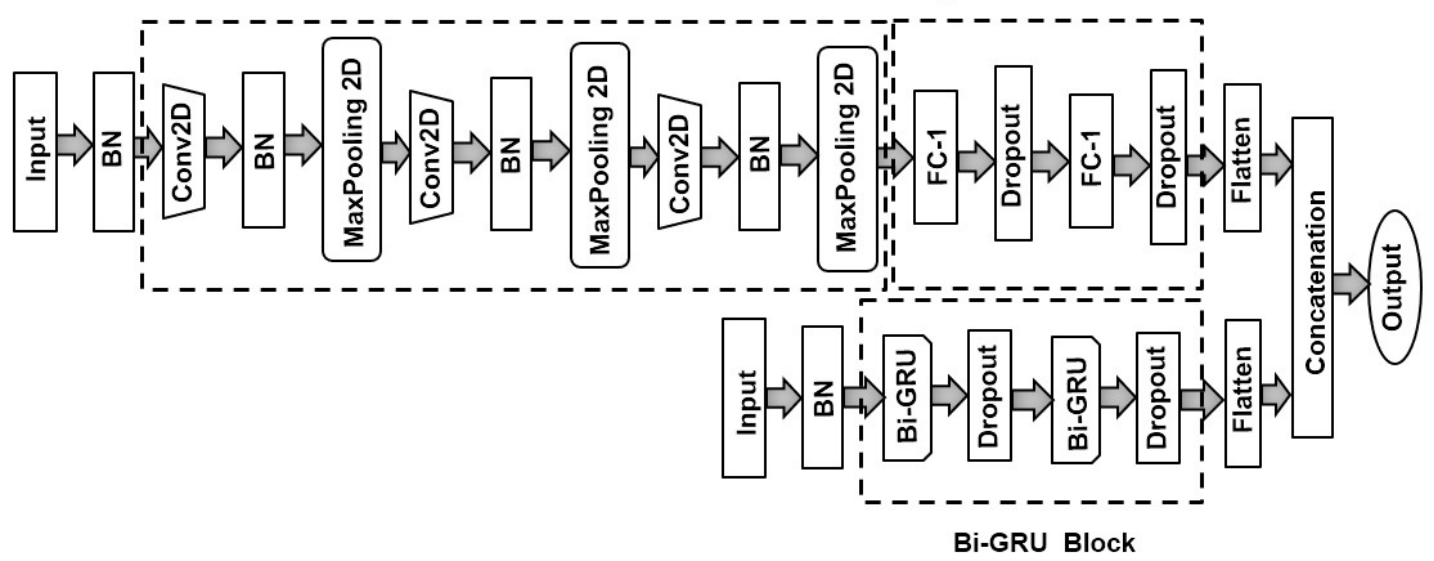

Fig. 6. Base Learner 4

2D Convolutional Block

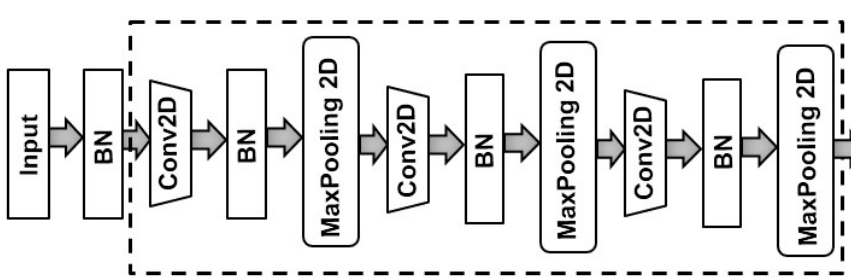

Bi-GRU Block

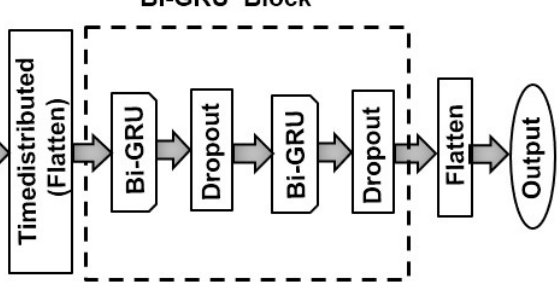

Fig. 7. Base Learner 5

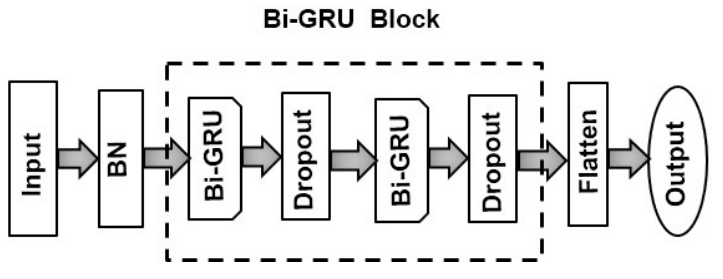

Fig. 8. Base Learner 2

after the 2D convolutional block to extract features further. Finally, we flattened the output and connected it to a dense layer for predicting the joint angles.

6) Fundamental Blocks: This subsection will discuss the fundamental blocks specifically designed to create effective base learners for our problem.

2D Convolutional Block. A 2D Convolutional Block consists of 2D convolutional, batch normalization (BN), and max-pooling layers. First, we have a $2 \mathrm{D}$ convolutional layer followed by a batch normalization layer. This batch normalization helps reduce the internal covariance shift of the network [34]. Internal covariance shift is the change in data distribution during model training as weights of the layers change during the training process. A max-pooling layer is then applied to reduce the feature space, reducing the complexity of the training [35]. Max-pooling also helps to select dominant convolved 
features to ensure efficient model learning. Convolutional, batch normalization, and max-pooling layers form the basic component of the 2D Convolutional block. This series of components are repeated serially three times to build the 2D convolutional block.

1D Convolutional Block. In a 1D convolutional block, two convolutional layers, a batch normalization layer, and a max-pooling layer are used as a basic building block. Two of these basic building block sequences are combined in series to construct the 1D convolutional block.

Bi-GRU Block. In bidirectional GRU, two GRU layers are trained simultaneously with input windows in both the positive and negative time directions [36]. As a result, bidirectional GRU can learn feature representation from both the past and the future for its predictions. This learning gives additional context to the network for training and often results in better model performance. Dropout layers were added after the Bi-GRU layers to avoid overfitting. A series of two Bi-GRU layers, each followed by a dropout layer, is used in the Bi-GRU block.

Fully Connected Block. A fully connected block consists of two fully connected dense layers (FC-1, FC-2). To avoid overfitting, a dropout layer was added after each fully connected dense layer.

\section{EXPERIMENTS}

\section{A. Data Collection}

Ten healthy subjects (six male and four female) participated in the study. All participants provided informed written consent before participating in the experiment. The Institutional Review Board (IRB) of the University of Central Florida (UCF) approved the study's protocol (IRB ID: STUDY00002011).

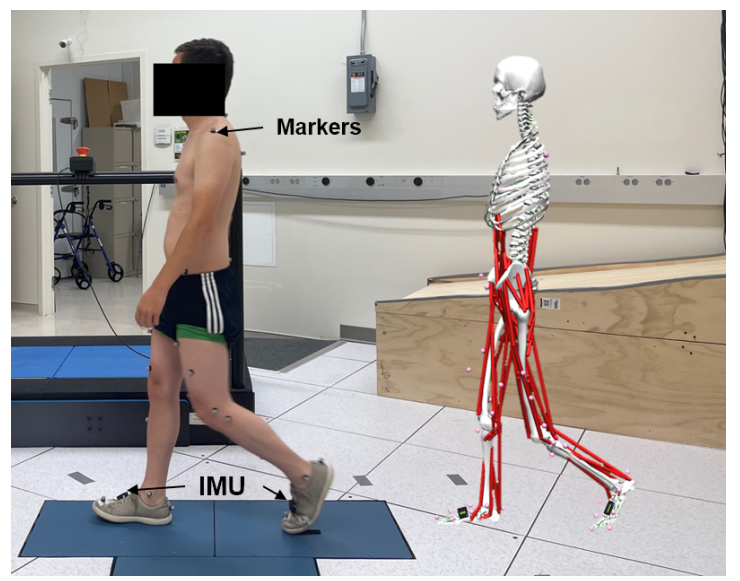

Fig. 9. Placement of Markers and IMU senors. Each IMU sensor was mounted on dorsum of foot. Musculoskeletal model was created using Scale and IK tool of OpenSim.

Two inertial measurement unit sensors were placed onto the participants' shoes (Fig.9). Each participant performed twelve trials: 4 treadmill, 4 overground, 2 stair, and 2 slope. Participants walked on a $5 \mathrm{~m}$ overground at four self-selected speeds,i.e., slow, normal, fast, and very fast. Each subject performed two trials on five-step stairs (27 inch wide, 9 inch deep, and 7 inch rise steps) with their self-selected speed. Participants also performed inclined and declined walking on a slope of 20\% [37], [38] at a self-selected speed. The participants walked on the treadmill at four different speeds for approximately 2 minutes. Non-dimensional slow, normal, fast, and very fast walking velocities were determined based on subject leg length, measured from the greater trochanter to the ground [39]. The actual treadmill speed was calculated by multiplying non-dimensional velocity with $\sqrt{g L_{l e g}}$, where $\mathrm{g}$ is the gravitational acceleration, and $L_{l e g}$ is the length of the participant's leg. During the trials, thirty-four reflective markers were placed on the participant (Fig. 9) based on a modified Helen-Hayes marker set [40]. Three-dimensional marker trajectories were captured with twelve infrared light cameras with a sampling rate of $100 \mathrm{~Hz}$ (Vicon, Oxford, UK). The accelerometer and gyroscope data were recorded with a sampling frequency of $\sim 148 \mathrm{~Hz}$ (Avanti wireless EMG, Delsys, Boston, MA). We used OpenSim [41], an open-source musculoskeletal analysis tool, to calculate joint angles during walking conditions. First, we scaled a generic musculoskeletal model while the participant held an anatomical pose. We performed inverse kinematics after scaling to estimate kinematics of the 23 coordinates of the musculoskeletal model for the dynamic walking trials. The sagittal plane angles of the hip, knee, and ankle for both legs, six joint angle coordinates, were the ground truth kinematics used in the training and validation of the machine learning models.

\begin{tabular}{cc}
\hline Condition & Trial Time (s) \\
\hline Treadmill & 5192.36 \\
Overground & 1683.78 \\
Slope Ascent & 767.48 \\
Slope Descent & 780.24 \\
Stair Ascent & 751.21 \\
Stair Descent & 662.12 \\
\hline \multicolumn{2}{c}{ TABLE I }
\end{tabular}

TOTAL TRIAL TIME FOR DIFFERENT WALKING CONDITION IN THE DATA SET

\section{B. Data Pre-processing}

Marker data collected from the motion capture system have a frequency of $100 \mathrm{~Hz}$, where IMU data were collected at a rate of $\sim 148 \mathrm{~Hz}$. IMU data were resampled to 100 samples per second to synchronize with the motion capture data before inputting to the machine learning model. In addition to the three-axis accelerometer and gyroscope data, we also calculated the norm of three-axis acceleration and angular rotation, resulting in eight features from each IMU sensor. We segmented the data into individual gait cycles for overground, stair, and slope trials, discarding the gait initialization phase. We allowed participants to initiate gait with either leg on the stair and slope trials. It will not introduce any bias to the model as we have sensors at both left and right feet. In Table I, we showed the total time for each walking condition.

The machine learning models considered a window length of 80 frames $(0.80 \mathrm{~s})$ as an input. We found that 80 frames of data gave optimal results for joint angle prediction. As the 80 frame input includes data from 6 joint angles, we have an output size of 480 for each 80 frame time interval.

One subject's data were set aside as the testing set for leave-outone subject cross-validation from the whole data set. The remainder of the data were split into a training set $(80 \%)$ for training the base learners and a validation set $(20 \%)$ to validate the model.

\section{Implementation Details}

1) DeepBBWAE-Net: Ten bootstrap samples $(\mathrm{k}=10)$ were generated for each BagBoost cell. We found that ten bootstrap samples work optimally for our problem. Bootstrap aggregation (bagging) is applied when the model becomes overfit, and significant variance is present in the data set. During training, the model may seem to fit the training data perfectly. When the model estimates the kinematics of a novel subject, it may not perform well due to high naturally occurring inter-subject gait pattern variability. So, indirectly the model has 
become overfit to the training data. As bagging is applied when the model becomes overfit and the data set has high variance, bagging can be an excellent approach to more accurate joint angle prediction. To further improve the generalization of joint angle prediction, we use GBM after bagging. Data used to validate the base models was utilized for training the gradient boosting model. Although we use dropout to avoid overfitting, the model had poor performance with the validation data compared to the training data. For this reason, to improve the generalization of the model, we used gradient boosting (GBM) techniques after the bagging prediction. We used the same methods after each base learner when training the base learners with the whole data set.

All the cells of the WAE block are generated using predictions from the leave-out-one subject cross-validation data. More specifically, we first estimated the kinematics of each subject with the BagBoost and BaseBoost blocks using leave-out-one cross-validation. We use the rest of the subjects' leave-out-one prediction data to find the optimal weights for each cell in the WAE block during test subject evaluation. We also compared the weighted average ensemble prediction of the base learners (named Base-WAE-Net) against our proposed method. We have used five base learners $(n=5)$ to create the DeepBBWAE-Net framework.

2) Base Learners: In this subsection, we will provide details on the implementation of the base learners.

Conv2D-Net. As a 2D convolutional layer requires a 4D tensor, we reshaped the 16 inputs of two sensors into 8 features $\times 2$ sensors. For three convolutional layers in the block, we used a filter size of 64,64 , and 128, respectively. The kernel size was $3 \times 3$ for all the convolutional layers. For maxpooling2D, we used a pool size of $2 \times 2$ for each layer. For the fully-connected block, we used a neuron count of 128 and 64 for two FC layers, respectively. A dropout rate of 0.5 was used for each dropout layer.

Bi-GRU-Net. Cell numbers 128 and 64 were used in each of the two Bi-GRU blocks. A dropout rate of 0.5 was used for each dropout layer.

Hybrid Conv1D-GRU-Net. The Bi-GRU block used the same parameters that were used in Bi-GRU-Net. For the $1 \mathrm{D}$ convolutional block, filter sizes of $64,64,128$, and 128 were used for four convolutional layers with a kernel size of 3 . A pool size of 2 was used for maxpooling1D. For a fully connected block, the neuron count was 128 and 64 for the dense layer, and a dropout rate was chosen 0.5 for both of the dropout layers.

Hybrid Conv2D-GRU-Net-1. The parameters for Hybrid Conv2DGRU-Net-1 were the same as those used in the respective components in base learners 1 and 2 .

Hybrid Conv2D-GRU-Net-2. We used the same parameters in Hybrid Conv2D-GRU-Net-1 as was used in base learners 1 and 2.

Hyperparameters of the Base Learner. We used mean squared error as the loss function and an Adam [42] optimizer to optimize the parameters of all the base learners. The batch size and iteration of all the base learners are provided in Table III.

\section{Evaluation Methods}

For the evaluation, we considered the leave-out-one subject crossvalidation method. Excluding a single subject's data from the training set allows for evaluation methods that check for model overfitting and assess the model's overall performance. To measure the accuracy of the predictions, we considered two metrics: Root Mean Square Error (RMSE) and Pearson correlation coefficient. RMSE determines the offset between the ground truth and the prediction, where the Pearson correlation coefficient determines the correlation of ground truth and predicted curves. To show the accuracy of our proposed approach, we take the average of angles of both legs to get single RMSE and correlation values for the hip, knee, and ankle angles on the sagittal plane under each walking test condition.

\section{E. Results}

Table II presents RSME and Pearson correlation coefficient values for all the base learners, intermediate models, and final proposed models for each joint angle under all walking conditions, as well as the overall mean values for each specific model. The models are categorized into five families: Base, BaseBoost, Bag, BagBoost, and WAE. To show the comparative performance of each family, we also reported the family means. We considered the five base models as the baseline for this study, so results from the base models are compared to intermediate and final proposed models to show the efficacy of the proposed methods.

While the Conv2D-Base-Net model had an RMSE of $6.62^{\circ}$ the use of boosting in the Base model, generating the Conv2D-BaseBoostNet model, resulted in a $4.38 \%$ reduction in mean RMSE. Applying bagging techniques on the training data resulted in a $2.11 \%$ reduction in mean RMSE compared to the Conv2D-Base-Net model. Using the same boosting methods on the validation data after bagging, as done in the Conv2D-BagBoost-Net model, reduced the mean RMSE by $7.10 \%$. Compared to the Bi-GRU-Base-Net model, a reduction in mean RMSE of $1.17 \%, 6.04 \%$, and $6.43 \%$ was found for Bi-GRU-BaseBoost-Net, Bi-GRU-Bag-Net, Bi-GRU-BagBoostNet, respectively. A mean RMSE reduction of $2.16 \%, 5.76 \%$, and 7.49\% was found with Hybrid Conv1D-GRU-BaseBoost-Net, Hybrid Conv1D-GRU-Bag-Net, and Hybrid Conv1D-GRU-BagBoostNet, respectively compared to the Hybrid Conv1D-GRU-Base-Net model. For the two remaining base models, mean RMSE reductions of $(1.19 \%, 6.14 \%$, and $6.34 \%)$ and $(2.12 \%, 7.35 \%$, and $8.01 \%)$ are found for the Bag, Boost, and BagBoost methods, respectively. The greatest mean RMSE reduction, $8.01 \%$, was found between the Hybrid Conv2D-GRU-BagBoost-Net-2 and its base equivalent. Similar trends are found in the Pearson correlation coefficient values when evaluating improvement with applying bagging and boosting to the base learners. These results demonstrate the effectiveness of adding different components to the base learners implemented in our proposed workflow.

After creating BaseBoost, Bag, and BagBoost models from the base learners, we used weighted average ensembles to combine those models. Base-WAE-Net, BaseBoost-WAE-Net, Bag-WAE-Net, and BagBoost-WAE-Net were created using the weighted average ensemble of the Base, BaseBoost, Bag, BagBoost family models, respectively. Base-WAE-Net, BaseBoost-WAE-Net, Bag-WAE-Net, and BagBoost-WAE-Net reduced the RMSE of the base models by $5.35 \%-27.79 \%, 5.74 \%-28.10 \%, 6.34 \%-28.55 \%$, and $6.53 \%-$ $28.70 \%$, respectively. Similarly, the weighted average ensemble of BaseBoost-WAE-Net, Bag-WAE-Net, and BagBoost-WAE-Net combined was used to create our proposed framework, DeepBBWAE-Net. DeepBBWAE-Net reduced the RMSE of baseline models by $6.93 \%$ $29.00 \%$. Pearson correlation coefficient increases of $0.60 \%-3.29 \%$, $0.64 \%-3.34 \%, 0.79 \%-3.49 \%, 0.81 \%-3.51 \%$, and $0.83 \%-3.53 \%$ from the base learners to Base-WAE-Net, BaseBoost-WAE-Net, BagWAE-Net, BagBoost-WAE-Net, and DeepBBWAE-Net respectively, were found.

The DeepBBWAE-Net model, compared with the BaseBoost, Bag, BagBoost, and other WAE family models, predicts kinematics with an RMSE reduction of $0.42 \%-27.47 \%$ and a Pearson correlation coefficient increase of $0.02 \%-3.38 \%$. Table II demonstrates the gradual improvements in both families mean RMSE and correlation values as bagging and boosting are added to the models. Overall, the WAE 


\begin{tabular}{|c|c|c|c|c|c|c|c|c|c|c|c|}
\hline \multirow[t]{2}{*}{ Family } & \multirow[t]{2}{*}{ Model } & \multicolumn{3}{|c|}{ RMSE } & \multirow[t]{2}{*}{ Mean } & \multirow{2}{*}{$\begin{array}{c}\text { Family } \\
\text { Mean }\end{array}$} & \multicolumn{3}{|c|}{ Pearson Corr. Coeff. } & \multirow[t]{2}{*}{ Mean } & \multirow{2}{*}{$\begin{array}{c}\text { Family } \\
\text { Mean }\end{array}$} \\
\hline & & Hip & Knee & Ankle & & & Hip & Knee & Ankle & & \\
\hline \multirow[t]{5}{*}{ Base } & Conv2D-Base-Net & 6.51 & 8.44 & 4.91 & 6.62 & 5.62 & 0.931 & 0.950 & 0.917 & 0.933 & 0.948 \\
\hline & Bi GRU-Base-Net & 5.23 & 6.07 & 4.09 & 5.13 & & 0.951 & 0.975 & 0.945 & 0.957 & \\
\hline & Hybrid Conv1D-GRU-Base-Net & 5.25 & 6.24 & 4.14 & 5.21 & & 0.951 & 0.974 & 0.943 & 0.956 & \\
\hline & Hybrid Conv2D-GRU-Base-Net-1 & 5.11 & 5.88 & 4.16 & 5.05 & & 0.952 & 0.975 & 0.947 & 0.958 & \\
\hline & Hybrid Conv2D-GRU-Base-Net-2 & 6.25 & 7.46 & 4.65 & 6.12 & & 0.934 & 0.957 & 0.922 & 0.938 & \\
\hline \multirow[t]{5}{*}{ Baseboost } & Conv2D-BaseBoost-Net & 6.47 & 7.77 & 4.75 & 6.33 & 5.49 & 0.932 & 0.952 & 0.919 & 0.934 & 0.949 \\
\hline & Bi GRU-BaseBoost-Net & 5.19 & 5.94 & 4.08 & 5.07 & & 0.953 & 0.975 & 0.946 & 0.958 & \\
\hline & Hybrid Conv1D-GRU-BaseBoost-Net & 5.26 & 5.94 & 4.09 & 5.10 & & 0.952 & 0.974 & 0.944 & 0.957 & \\
\hline & Hybrid Conv2D-GRU-BaseBoost-Net-1 & 5.15 & 5.78 & 4.03 & 4.99 & & 0.953 & 0.976 & 0.948 & 0.959 & \\
\hline & Hybrid Conv2D-GRU-BaseBoost-Net-2 & 6.19 & 7.21 & 4.57 & 5.99 & & 0.934 & 0.957 & 0.924 & 0.939 & \\
\hline \multirow[t]{5}{*}{ Bag } & Conv2D-Bag-Net & 6.51 & 8.20 & 4.73 & 6.48 & 5.32 & 0.937 & 0.954 & 0.927 & 0.939 & 0.956 \\
\hline & Bi GRU-Bag-Net & 4.99 & 5.57 & 3.89 & 4.82 & & 0.959 & 0.978 & 0.954 & 0.964 & \\
\hline & Hybrid Conv1D-GRU-Bag-Net & 4.95 & 5.84 & 3.94 & 4.91 & & 0.960 & 0.978 & 0.951 & 0.963 & \\
\hline & Hybrid Conv2D-GRU-Bag-Net-1 & 4.91 & 5.48 & 3.83 & 4.74 & & 0.961 & 0.979 & 0.956 & 0.966 & \\
\hline & Hybrid Conv2D-GRU-Bag-Net-2 & 5.92 & 6.79 & 4.30 & 5.67 & & 0.944 & 0.964 & 0.936 & 0.948 & \\
\hline \multirow[t]{5}{*}{ Bagboost } & Conv2D-BagBoost-Net & 6.40 & 7.53 & 4.54 & 6.15 & 5.22 & 0.938 & 0.956 & 0.929 & 0.941 & 0.956 \\
\hline & Bi GRU-BagBoost-Net & 4.97 & 5.56 & 3.87 & 4.80 & & 0.959 & 0.978 & 0.955 & 0.964 & \\
\hline & Hybrid Conv1D-GRU-BagBoost-Net & 4.97 & 5.58 & 3.90 & 4.82 & & 0.960 & 0.978 & 0.952 & 0.963 & \\
\hline & Hybrid Conv2D-GRU-BagBoost-Net-1 & 4.93 & 5.45 & 3.80 & 4.73 & & 0.961 & 0.979 & 0.956 & 0.966 & \\
\hline & Hybrid Conv2D-GRU-BagBoost-Net-2 & 5.89 & 6.73 & 4.27 & 5.63 & & 0.945 & 0.964 & 0.936 & 0.948 & \\
\hline \multirow[t]{5}{*}{ WAE } & Base-WAE-Net & 4.89 & 5.60 & 3.86 & 4.78 & 4.74 & 0.959 & 0.978 & 0.954 & 0.964 & 0.965 \\
\hline & BaseBoost-WAE-Net & 4.96 & 5.51 & 3.82 & 4.76 & & 0.959 & 0.979 & 0.955 & 0.964 & \\
\hline & Bag-WAE-Net & 4.92 & 5.48 & 3.77 & 4.73 & & 0.961 & 0.979 & 0.956 & 0.966 & \\
\hline & BagBoost-WAE-Net & 4.93 & 5.45 & 3.76 & 4.72 & & 0.961 & 0.979 & 0.957 & 0.966 & \\
\hline & DeepBBWAE-Net & 4.90 & 5.44 & 3.76 & 4.70 & & 0.961 & 0.979 & 0.957 & 0.966 & \\
\hline
\end{tabular}

TABLE II

RMSE AND PEARSON CORRELATION COEFFICIENT OF HIP. KNEE, AND ANKLE ANGLE FOR ALL MODELS FOR ALL WALKING SCENARIOS

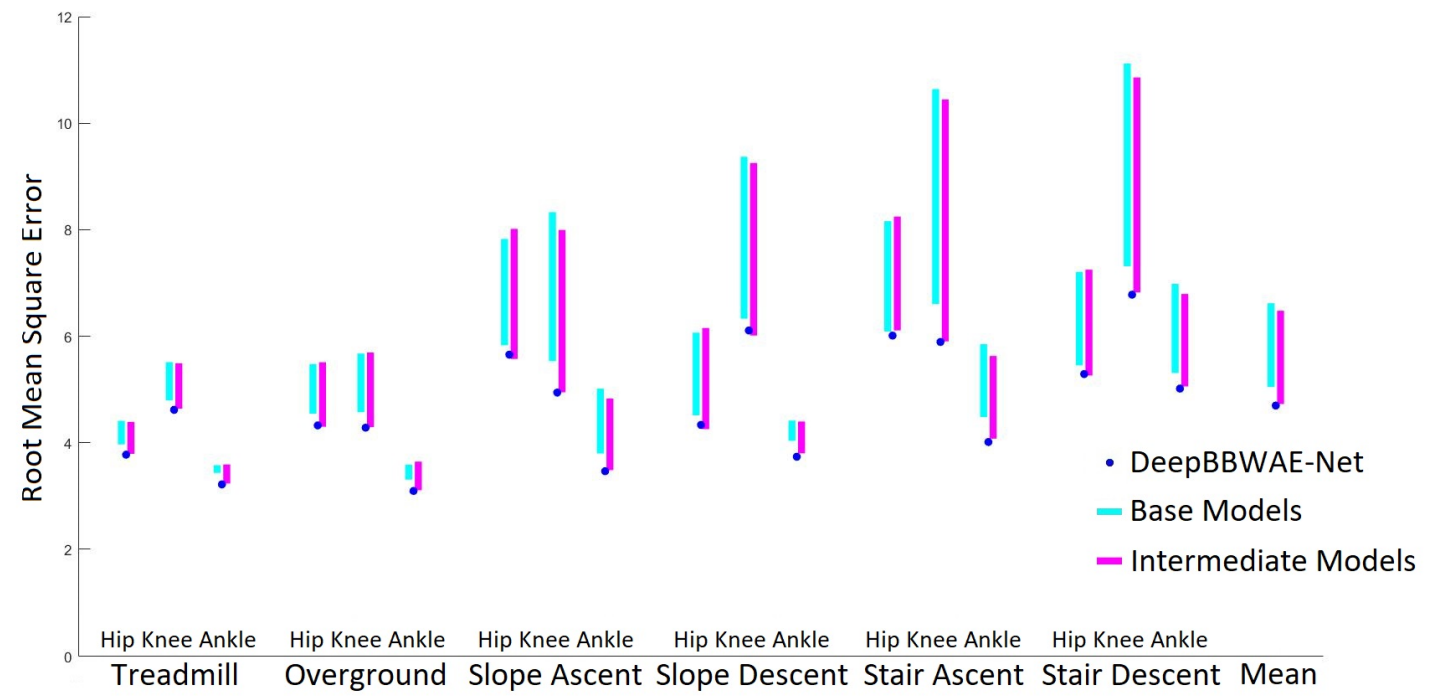

Fig. 10. Range of RMSE plot for Base models, Intermediate models, and DeepBBWAE-Net

\begin{tabular}{ccc}
\hline Base Learners & Batch Size & Iteration \\
\hline Conv2D-Net & 64 & 250 \\
Bi-GRU-Net & 64 & 70 \\
Hybrid Conv1D-GRU-Base-Net & 64 & 200 \\
Hybrid Conv2D-GRU-Base-Net-1 & 64 & 200 \\
Hybrid Conv2D-GRU-Base-Net-2 & 64 & 70 \\
\hline
\end{tabular}

TABLE III

HYPERPARAMETERS OF THE BASE MODELS

family reduces the mean RMSE by $15.66 \%, 13.66 \%, 10.90 \%$, and 9.20\% compared to the Base, BaseBoost, Bag, BagBoost families, respectively while increasing the Pearson correlation coefficients by
$1.76 \%, 1.66 \%, 0.95 \%$, and $0.90 \%$ respectively.

In Fig. 10, 11, we compared the ranges in RMSE and Pearson correlation coefficients of the base and intermediate models (the BaseBoost, Bag, and BagBoost models) with our proposed DeepBBWAENet. For both RMSE and correlation, the base and intermediate model ranges were wider in the slope and stair conditions but more narrow for the treadmill and overground conditions.

Table IV demonstrates the mean RMSE and Pearson correlation coefficients of the base models, the intermediate models, and DeepBBWAE-Net for different walking conditions. Compared to the base models, the intermediate models reduced the RMSE by $4.77 \%, 5.49 \%, 8.29 \%, 7.52 \%, 8.84 \%$, and $6.98 \%$ for the treadmill, 


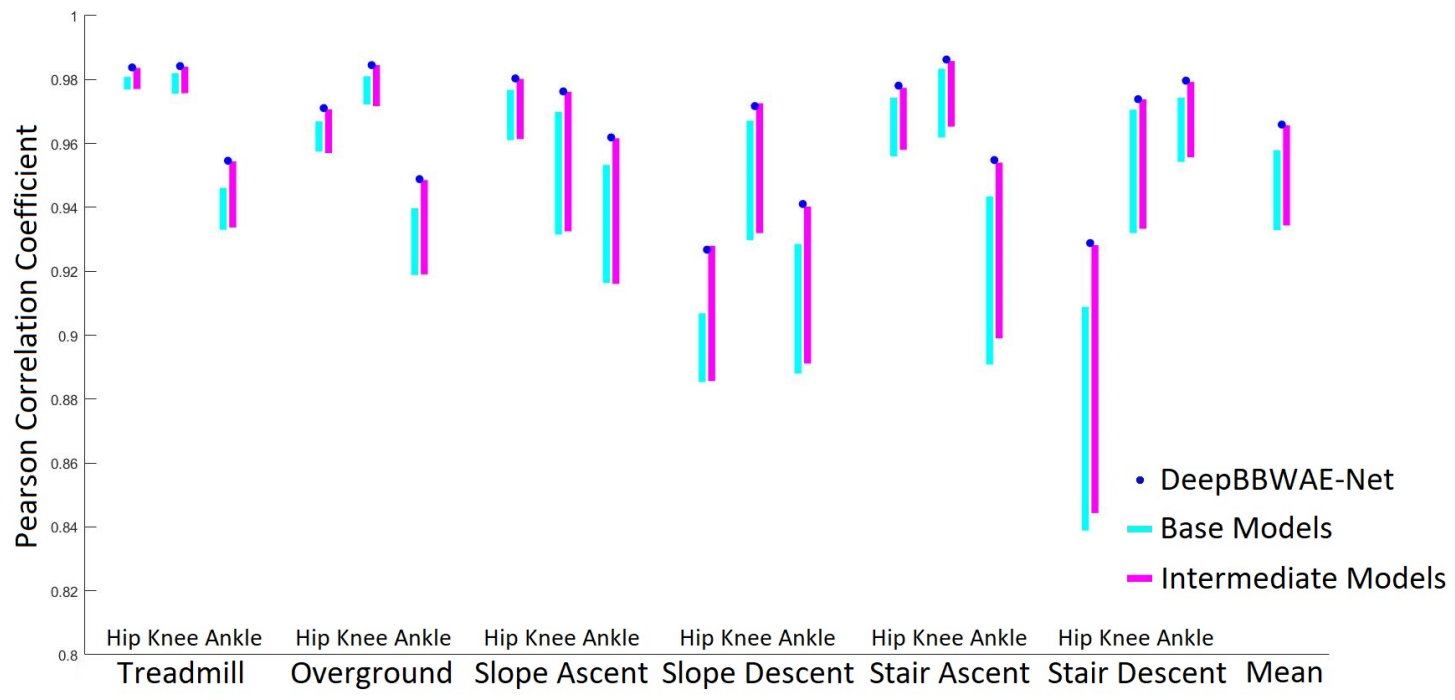

Fig. 11. Range of Pearson Correlation Coefficient plot for Base models, Intermediate models, and DeepBBWAE-Net

\begin{tabular}{cccccccc}
\hline & Model & Treadmill & Overground & Slope ascent & Slope descent & Stair ascent & Stair descent \\
\hline \multirow{2}{*}{ RMSE } & Base Models & 4.29 & 4.48 & 5.81 & 5.68 & 6.57 & 6.92 \\
& Intermediate Models & 4.09 & 4.23 & 5.33 & 5.25 & 5.99 & 6.44 \\
& DeepBBWAE-Net & 3.87 & 3.90 & 4.69 & 4.73 & 5.31 & 5.69 \\
\hline Correlation & Base Models & 0.966 & 0.957 & 0.955 & 0.922 & 0.955 & 0.935 \\
& Intermediate Models & 0.970 & 0.962 & 0.962 & 0.933 & 0.964 & 0.946 \\
& DeepBBWAE-Net & 0.974 & 0.968 & 0.973 & 0.946 & 0.973 & 0.961 \\
\hline
\end{tabular}

TABLE IV

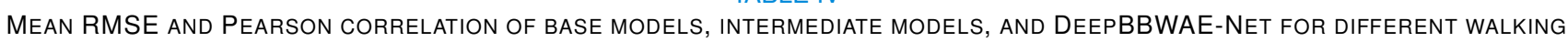
CONDITION

overground, slope ascent, slope descent, stair ascent, and stair descent conditions, respectively. DeepBBWAE-Net reduced the RMSE by $9.82 \%, 12.94 \%, 19.30 \%, 16.76 \%, 19.25 \%$, and $17.72 \%$ for the same conditions. In terms of Pearson correlation coefficients, the intermediate models saw an increase in correlation of $0.41 \%, 0.54 \%$, $0.78 \%, 1.18 \%, 0.90 \%$, and $1.20 \%$ while DeepBBWAE-Net had an increase of $0.82 \%, 1.19 \%, 1.89 \%, 2.62 \%, 1.89 \%$, and $2.78 \%$ for the six walking conditions.

\section{DIscussion}

A Novel DeepBBWAE-Net. This paper proposes a novel framework, DeepBBWAE-Net, by implementing bagging, boosting, and weighted average ensemble techniques to estimate joint angles of the lower extremities during gait in different environments using two IMU sensors. The reduced RMSE indicates DeepBBWAE-Net's ability to calculate joint kinematics more accurately than other simpler deep learning models. Our results demonstrate that walking conditions with significantly more variability lead to predictions with greater RMSE and lower Pearson correlation coefficients. For example, the joint kinematics of walking is highly dependent on walking speed [43]. The treadmill trials had a controlled constant walking speed throughout the data collection period. On the other hand, when the participants walked back and forth across 5 meter walkways for the overground and sloped walking conditions, there was significantly more variation in walking speed leading to increased joint kinematic variability. Similarly, The variety of walking strategies for stair walking led to predictions with higher RMSEs and lower Pearson correlation coefficients than those of kinematic predictions of other walking conditions. However, DeepBBAWE-Net achieved better joint kinematic predictions than conventional deep learning models under these more variable walking patterns.

Stair and Slope Kinematics. Many studies have implemented machine learning to estimate kinematics using IMU sensors [13], [15]-[17] for overground or treadmill conditions. But, no study has developed machine learning-based estimation of joint kinematics for stair and slope walking. Our final goal is to estimate joint kinematics outside the lab in different walking environments. Training an algorithm with data from stair and slope conditions is a big step toward achieving that goal.

Predictions of the treadmill and overground conditions had lower RMSEs, while the slope and stair condition predictions produced significantly more error. One reason for this is the greater number of consecutive data sets under each controlled walking speed for the treadmill and overground conditions compared to the slope and stair conditions. This is further compounded by the fact that the stair and slope trials have greater kinematic variability. These variances in the kinematics and reduced data set size makes determining the specific window size for input data to the algorithm difficult.

Despite new data management and our new algorithm, the stair condition predictions had greater errors than other walking conditions. The increased error is likely due to the variations in strategies we observed with subjects in the stair walking condition. We found that some participants used their forefoot to climb up the stairs while other participants used their entire foot. Some participants contacted their toe first while descending the stairs, while other participants 
used their whole foot to land on the stair.

Real-time measurement. One of the advantages of our study is the potential for estimation of joint kinematics in real-time as raw IMU data is used as input to the model. Previous studies would typically pre-process the collected sensor signal and motion data before input to a machine learning algorithm. For example, gait cycles would be identified and then used to normalized the time data to unify the input data size [13], [20]. Also, input features are usually extracted from the collected data to use as algorithm input [20]. This pre-processing increases computation time, slowing the predictions and reducing the network's viability as a real-time system, preventing the monitoring of kinematics in real-time. Our proposed model uses raw IMU data, removing the need for computationally expensive pre-processing and enabling application in real-time kinematic estimation.

Comparison with Other Studies. Direct comparison of this study's results with other studies must be made in light of the differences in data set size, sensor count, and trial conditions. The data set used in this study consisted of a combination of treadmill, overground, stair, and slope walking data. While perhaps a more robust representation of human gait, using a combination of different conditions may produce less accurate predictions than a model trained to predict kinematics from the treadmill or overground data only. For this reason, we compare the results of our proposed approach with more conventional models applied to the same data set. Other studies use conventional machine learning or deep learning models (artificial neural networks [20], single LSTM layer [16], 1D convolutional neural networks [21], or 2D convolutional neural networks [17]) to predict joint kinematics in gait. Our proposed method outperforms conventional deep learning techniques significantly when trained from the same data set. This result indicates the advantages of our developed machine learning algorithm over those of other studies to predict joint kinematics in gait more robustly.

Limitation and Future Work. One of the significant challenges addressed in this study is the development of a kinematics estimation approach that requires a reduced number of sensors. It is challenging because the algorithm's input is missing information typically captured with a complete sensor set. This can potentially affect the model's accuracy. While adding some sensors onto the thigh or shank may improve the performance of kinematics estimation, it makes the system less practical due to the potential burden on the subject. Another limitation of our work is the limited data set. A larger data set would more effectively train the model and result in better kinematic predictions. One potential method for expanding the available data set is to use simulated IMU sensor data [15]. This would be particularly useful for expanding areas of the data set that were less represented, such as stair and slope walking conditions [44]. Using simulation to generate data of hundreds of different artificial subjects may improve the generalization of DeepBBWAE-Net. In the future, we plan to add simulated data to the training and assess the model's performance with the augmented data set.

\section{CONCLUSION}

In this paper, we proposed a novel deep learning framework DeepBBWAE-Net to estimate lower extremity kinematics in different walking conditions. Our proposed machine learning model predicts joint kinematics with significantly less error compared to other conventional deep learning models. This study implements a reduced set of IMU sensors in order to increase the system's viability in commercial systems by increasing user comfort. This method shows potential for applications in continuous gait monitoring in the realworld.

\section{REFERENCES}

[1] K. Delbaere, D. L. Sturnieks, G. Crombez, and S. R. Lord, "Concern About Falls Elicits Changes in Gait Parameters in Conditions of Postural Threat in Older People," The Journals of Gerontology: Series A, vol. 64A, no. 2, pp. 237-242, 02 2009. [Online]. Available: https://doi.org/10.1093/gerona/gln014

[2] K. R. Pirker W, "Gait disorders in adults and the elderly : A clinical guide." Wien Klin Wochenschr, vol. 129, no. 3-4, pp. 81-95, 2017.

[3] F. A. Fatoye, M. L. van der Linden, P. J. Rowe, S. Palmer, and F. Macmillan, "Gait kinematics and passive knee joint range of motion in children with hypermobility syndrome," Gait and Posture, vol. 33, pp. 447-451, 2011.

[4] K.-S. Shih, S.-Y. Jhou, W.-C. Hsu, C.-C. Hsu, J.-W. Chen, J.-C. Yeh, and Y.-C. Hung, "A biomechanical investigation of athletic footwear traction performance: Integration of gait analysis with computational simulation," Applied Sciences, vol. 10, no. 5, 2020. [Online]. Available: https://www.mdpi.com/2076-3417/10/5/1672

[5] B. R., "Gait analysis methods in rehabilitation," Journal of neuroengineering and rehabilitation, vol. 3:4, 2006.

[6] I. Weygers, M. Kok, M. Konings, H. Hallez, H. De Vroey, and K. Claeys, "Inertial sensor-based lower limb joint kinematics: A methodological systematic review," Sensors, vol. 20, no. 3, 2020.

[7] E. Palermo, S. Rossi, F. Marini, F. Patanè, and P. Cappa, "Experimental evaluation of accuracy and repeatability of a novel body-to-sensor calibration procedure for inertial sensor-based gait analysis," Measurement, vol. 52, pp. 145-155, 2014.

[8] T. Zimmermann, B. Taetz, and G. Bleser, "Imu-to-segment assignment and orientation alignment for the lower body using deep learning," Sensors, vol. 18, no. 1, 2018.

[9] G. Mayer-Kress, Y.-T. Liu, and K. M. Newell, "Complex systems and human movement," Complexity, vol. 12, no. 2, pp. 40-51, 2006. [Online]. Available: https://onlinelibrary.wiley.com/doi/abs/10.1002/cplx.20151

[10] J. C. Alcaraz, S. Moghaddamnia, M. Fuhrwerk, and J. Peissig, "Efficiency of the memory polynomial model in realizing digital twins for gait assessment," in 2019 27th European Signal Processing Conference (EUSIPCO), 2019, pp. 1-5.

[11] A. Baghdadi, L. A. Cavuoto, and J. L. Crassidis, "Hip and trunk kinematics estimation in gait through kalman filter using imu data at the ankle," IEEE Sensors Journal, vol. 18, no. 10, pp. 4253-4260, 2018.

[12] A. Salarian, P. R. Burkhard, F. J. G. Vingerhoets, B. M. Jolles, and K. Aminian, "A novel approach to reducing number of sensing units for wearable gait analysis systems," IEEE Transactions on Biomedical Engineering, vol. 60, no. 1, pp. 72-77, 2013.

[13] M. Mundt, A. Koeppe, S. David, T. Witter, F. Bamer, W. Potthast, and B. Markert, "Estimation of gait mechanics based on simulated and measured imu data using an artificial neural network," Frontiers in Bioengineering and Biotechnology, vol. 8, p. 41, 2020.

[14] C.Maiwald, T. T. Mayer, and T.L.Milani, "Detecting foot-to-ground contact from kinematic data in running," Footwear Science, vol. 1, no. 2, pp. 111-118, 2009 .

[15] M. Mundt, A. Koeppe, F. Bamer, S. David, and B. Markert, "Artificial neural networks in motion analysis-applications of unsupervised and heuristic feature selection techniques," Sensors, vol. 20, no. 16, 2020. [Online]. Available: https://www.mdpi.com/1424-8220/20/16/4581

[16] M. Mundt, W. Thomsen, T. Witter, A. Koeppe, S. David, F. Bamer, W. Potthast, and B. Markert, "Prediction of lower limb joint angles and moments during gait using artificial neural networks," Medical amp; biological engineering amp; computing, vol. 58, no. 1, p. 211-225, January 2020. [Online]. Available: https://doi.org/10.1007/s11517-01902061-3

[17] E. Dorschky, M. Nitschke, C. F. Martindale, A. J. van den Bogert, A. D. Koelewijn, and B. M. Eskofier, "Cnn-based estimation of sagittal plane walking and running biomechanics from measured and simulated inertial sensor data," Frontiers in Bioengineering and Biotechnology, vol. 8, p. 604, 2020. [Online]. Available: https://www.frontiersin.org/article/10.3389/fbioe.2020.00604

[18] V. Hernandez, D. Dadkhah, V. Babakeshizadeh, and D. Kulić, "Lower body kinematics estimation from wearable sensors for walking and running: A deep learning approach," Gait Posture, vol. 83, pp. 185-193, 2021. [Online]. Available: https://www.sciencedirect.com/science/article/pii/S0966636220306068

[19] E. Rapp, S. Shin, W. Thomsen, R. Ferber, and E. Halilaj, "Estimation of kinematics from inertial measurement units using a combined deep learning and optimization framework," Journal of Biomechanics, vol. 116, p. 110229, 2021. [Online]. Available: https://www.sciencedirect.com/science/article/pii/S0021929021000099 
[20] H. Lim, B. Kim, and S. Park, "Prediction of lower limb kinetics and kinematics during walking by a single imu on the lower back using machine learning," Sensors, vol. 20, no. 1, 2020.

[21] M. Gholami, C. Napier, and C. Menon, "Estimating lower extremity running gait kinematics with a single accelerometer: A deep learning approach," Sensors, vol. 20, no. 10, 2020. [Online]. Available: https://www.mdpi.com/1424-8220/20/10/2939

[22] M. S. . P. J. Conte Alcaraz, J., "Efficiency of deep neural networks for joint angle modeling in digital gait assessment," EURASIP J. Adv. Signal Process, vol. 10 (2021), 2021.

[23] S. Hochreiter and J. Schmidhuber, "Long short-term memory," Neural Comput., vol. 9, no. 8, p. 1735-1780, Nov. 1997. [Online]. Available: https://doi.org/10.1162/neco.1997.9.8.1735

[24] K. Cho, B. van Merrienboer, C. Gulcehre, D. Bahdanau, F. Bougares, H. Schwenk, and Y. Bengio, "Learning phrase representations using rnn encoder-decoder for statistical machine translation," 2014.

[25] M. Gadaleta and M. Rossi, "Idnet: Smartphone-based gait recognition with convolutional neural networks," Pattern Recognition, vol. 74, pp. 25-37, 2018. [Online]. Available: https://www.sciencedirect.com/science/article/pii/S0031320317303485

[26] R. Delgado-Escaño, F. M. Castro, J. R. Cózar, M. J. Marín-Jiménez, and N. Guil, "An end-to-end multi-task and fusion cnn for inertial-based gait recognition," IEEE Access, vol. 7, pp. 1897-1908, 2019.

[27] L. Breiman, "Bagging predictors," Mach. Learn., vol. 24 no. 2, p. 123-140, Aug. 1996. [Online]. Available: https://doi.org/10.1023/A:1018054314350

[28] J. H. Friedman, "Greedy function approximation: A gradient boosting machine." The Annals of Statistics, vol. 29, no. 5, pp. 1189 - 1232, 2001.

[29] K. S. D Kraft, "SLSQP - a nonlinear programming method with quadratic programming subproblems," DLR, Oberpfaffenhofen, 1989.

[30] C. L. Lawson and R. J. Hanson, Solving Least Squares Problems. SIAM, 1974, vol. 161.

[31] R. Fletcher, Practical Methods of Optimization; (2nd Ed.). USA: WileyInterscience, 1987.

[32] F. Li, K. Shirahama, M. A. Nisar, X. Huang, and M. Grzegorzek, "Deep transfer learning for time series data based on sensor modality classification," Sensors, vol. 20, no. 15, 2020. [Online]. Available: https://www.mdpi.com/1424-8220/20/15/4271

[33] F. Chollet et al. (2015) Keras. [Online]. Available: https://github.com/fchollet/keras

[34] S. Ioffe and C. Szegedy, "Batch normalization: Accelerating deep network training by reducing internal covariate shift," 2015.

[35] D. Scherer, A. Müller, and S. Behnke, "Evaluation of pooling operations in convolutional architectures for object recognition," in Artificial Neural Networks - ICANN 2010, K. Diamantaras, W. Duch, and L. S. Iliadis, Eds. Berlin, Heidelberg: Springer Berlin Heidelberg, 2010, pp. 92-101.

[36] M. Schuster and K. K. Paliwal, "Bidirectional recurrent neural networks," IEEE Transactions on Signal Processing, vol. 45, no. 11, pp. 2673-2681, 1997.

[37] K. Karamanidis and A. Arampatzis, "Evidence of mechanical load redistribution at the knee joint in the elderly when ascending stairs and ramps," Annals of biomedical engineering, vol. 37, no. 3, p. 467-476, March 2009.

[38] I. Komnik, M. Peters, J. Funken, S. David, S. Weiss, and W. Potthast, "Non-sagittal knee joint kinematics and kinetics during gait on level and sloped grounds with unicompartmental and total knee arthroplasty patients," PLOS ONE, vol. 11, no. 12, pp. 1-18, 12 2016. [Online]. Available: https://doi.org/10.1371/journal.pone.0168566

[39] M. Q. Liu, F. C. Anderson, M. H. Schwartz, and S. L. Delp, "Muscle contributions to support and progression over a range of walking speeds," Journal of Biomechanics, vol. 41, no. 15, pp. 3243-3252, 2008.

[40] R. H. K. . W. M. E. Kadaba, M. P., "Measurement of lower extremity kinematics during level walking," Journal of Orthopaedic Research : Official Publication of the Orthopaedic Research Society, vol. 8, no. 3, pp. 383-392, 1990.

[41] S. L. Delp, F. C. Anderson, A. S. Arnold, P. Loan, A. Habib, C. T. John, E. Guendelman, and D. G. Thelen, "Opensim: Open-source software to create and analyze dynamic simulations of movement," IEEE Transactions on Biomedical Engineering, vol. 54, no. 11, pp. 1940-1950, 2007.

[42] D. P. Kingma and J. Ba, "Adam: A method for stochastic optimization," 2017.

[43] M. H. Schwartz, A. Rozumalski, and J. P. Trost, "The effect of walking speed on the gait of typically developing children," Journal of Biomechanics, vol. 41, no. 8, pp. 1639-1650, 2008. [Online]. Available: https://www.sciencedirect.com/science/article/pii/S0021929008001450
[44] T. Lencioni, I. Carpinella, M. Rabuffetti, A. Marzegan, and M. Ferrarin, "Human kinematic, kinetic and emg data during different walking and stair ascending and descending tasks," Scientific data, vol. 6, no. 1, Dec. 2019. 